ARTICLE

Received 29 Mar 2016 | Accepted 22 Sep 2016 | Published 11 Nov 2016

DOI: $10.1038 /$ ncomms13323

OPEN

\title{
Biocatalytic trifluoromethylation of unprotected phenols
}

\author{
Robert C. Simon ${ }^{1}$, Eduardo Busto ${ }^{1}$, Nina Richter ${ }^{2}$, Verena Resch ${ }^{1}$, Kendall N. Houk ${ }^{3} \&$ Wolfgang Kroutil ${ }^{1}$
}

Organofluorine compounds have become important building blocks for a broad range of advanced materials, polymers, agrochemicals, and increasingly for pharmaceuticals. Despite tremendous progress within the area of fluorination chemistry, methods for the direct introduction of fluoroalkyl-groups into organic molecules without prefunctionalization are still highly desired. Here we present a concept for the introduction of the trifluoromethyl group into unprotected phenols by employing a biocatalyst (laccase), $\mathrm{tBuOOH}$, and either the Langlois' reagent or Baran's zinc sulfinate. The method relies on the recombination of two radical species, namely, the phenol radical cation generated directly by the laccase and the $\mathrm{CF}_{3}$-radical. Various functional groups such as ketone, ester, aldehyde, ether and nitrile are tolerated. This laccase-catalysed trifluoromethylation proceeds under mild conditions and allows accessing trifluoromethyl-substituted phenols that were not available by classical methods.

\footnotetext{
${ }^{1}$ Department of Chemistry, Organic and Bioorganic Chemistry, University of Graz, NAWI Graz, BioTechMed Graz, Heinrichstrasse 28, 8010-Graz, Austria. ${ }^{2}$ ACIB GmbH, c/o Heinrichstrasse 28, 8010-Graz, Austria. ${ }^{3}$ Department of Chemistry and Biochemistry, University of California, Los Angeles, California 90095, USA. Correspondence and requests for materials should be addressed to R.C.S. (email: robert.simon@gmx.net) or to K.N.H. (email: houk@chem.ucla.edu) or to W.K. (email: wolfgang.kroutil@uni-graz.at).
} 
T he introduction of fluoroalkyl-groups (for example, $\mathrm{CF}_{3}$, $\mathrm{CHF}_{2}, \mathrm{CH}_{2} \mathrm{~F}$, etc.) into organic compounds has become a major subject in various fields of chemical research, in particular medicinal chemistry and drug discovery ${ }^{1,2}$. This is due to the metabolic stability, increased permeability or enhanced binding properties of the organo-fluorine compounds in comparison to their non-fluorinated counterparts ${ }^{3}$. Among all fluorine-containing moieties, the trifluoromethyl group is privileged $^{4}$, and trifluoromethylated arenes are of interest for agrochemicals, pharmaceuticals and advanced materials ${ }^{5}$. Several excellent methods to provide structurally diverse $\mathrm{CF}_{3^{-}}$building blocks have been elaborated ${ }^{6-8}$ : common strategies to introduce the $\mathrm{CF}_{3}$-group into aromatic compounds involve metal-mediated/catalysed functional group interconversions $s^{9,10}$ where halogens $s^{11-13}$, boronic acids ${ }^{4-16}$, boronates ${ }^{17,18}$ and even amines ${ }^{19,20}$ are replaced by nucleophilic, electrophilic or radical $\mathrm{CF}_{3}$-sources (Fig. 1a). Other methods rely on directing groups ${ }^{21-23}$, as well as on visible light and photo-catalysis (Fig. $1 \mathrm{~b}, \mathrm{c})^{24-26}$.

While trifluoromethylation of (substituted) mono- and biaryl-systems has been broadly investigated ${ }^{27-30}$, only few reports deal with the transformation of unprotected phenols (Fig. 1d $)^{31-33}$, and these give non-regioselective transformations and/or unsatisfying conversions. Hence, a general method for attaching the $\mathrm{CF}_{3}$-moiety to phenols in a practical manner remains elusive. We report an efficient and selective method for trifluoromethylation of unprotected phenols by biocatalytic introduction of a trifluoromethyl group derived from common precursors.

\section{Results}

Reaction concept. The approach for the trifluoromethylation of phenols via $\mathrm{C}-\mathrm{C}$ bond formation presented in this paper is based on the recombination of two radicals, namely a $\mathrm{CF}_{3}$-radical and a phenol-derived radical, wherein the two radicals are formed via two different pathways (Fig. 2). The phenol-derived radical is formed by a laccase (E.C. 1.10.3.2), which catalyses in general the one-electron oxidation of phenols and anilines using molecular oxygen as the oxidant ${ }^{34-38}$. Simultaneously, the electrophilic $\mathrm{CF}_{3}$-radical is generated in situ from either Langlois' reagent $\left(\mathrm{NaSO}_{2} \mathrm{CF}_{3}\right)^{31,39,40}$ or Baran's zinc sulfinate

a

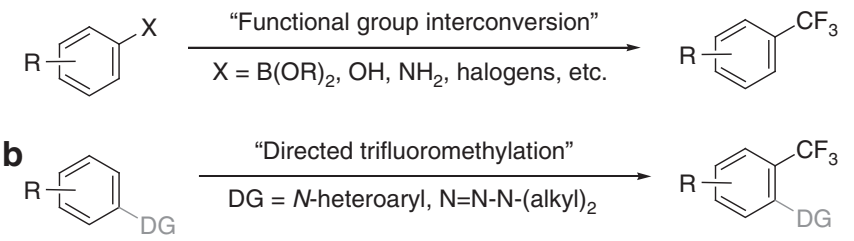

C

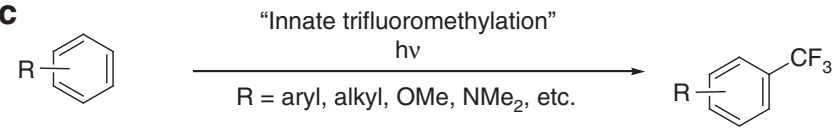

d

This work
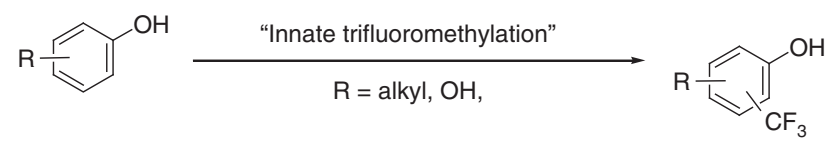

Figure 1 | Methods to attach the $\mathbf{C F}_{\mathbf{3}}$-group to arenes. (a) Functional group interconversion; (b) Trifluoromethylation controlled by a directing group (DG); (c) Innate trifluoromethylation of substituted arenes, accept phenols; (d) Innate trifluoromethylation of phenols.
$\left(\mathrm{Zn}\left(\mathrm{SO}_{2} \mathrm{CF}_{3}\right)_{2}=\mathrm{TFMS}\right)^{41}$. The $\mathrm{CF}_{3}$-radical may also be formed under electrochemical conditions from TFMS ${ }^{42}$.

The concept was initially tested by employing the laccase from Agaricus bisporus and using the electron-rich phenol 1a as substrate and TFMS as trifluoromethylation agent with tert-butyl hydroperoxide $(t \mathrm{BuOOH})$ as the oxidant. This ortho- and para-substituted substrate was chosen to minimize literatureknown fast di- or oligomerization in ortho and para positions initiated by laccases ${ }^{34-37,43}$. The chemical-enzymatic system led to successful product formation, whereby O-alkylation was not detected, but $\mathrm{C}-\mathrm{C}$ bond formation at the free $\mathrm{C}-\mathrm{H}$ of the arene was observed, leading to product $2 \mathrm{a}$ with $58 \%$ of the transformed substrate (Table 1, entry 1).

The phenol did not react in the absence of laccase and in the presence of TFMS and $t \mathrm{BuOOH}$ (Entry 2). In the presence of the laccase but in the absence of TFMS/ $t \mathrm{BuOOH}$, substrate 1a was just di-/polymerized (Entry 3). Also, performing the reaction with laccase and TFMS in the absence of $t \mathrm{BuOOH}$ did not lead to the desired product formation of $\mathbf{2 a}$, indicating that $t \mathrm{BuOOH}$ is required to form the $\mathrm{CF}_{3}$-radical (Entry 4). Thus, product $2 \mathrm{a}$ was only formed in the presence of laccase and TFMS $/ t \mathrm{BuOOH}$. $t \mathrm{BuOOH}$ cannot be substituted by hydrogen peroxide, since in this case neither the formation of trifluoromethylated 2a nor any other transformation of 1a was observed (entry 5).

Encouraged by this initial result, the radical trifluoromethylation of 1a was optimized, testing varied concentrations of TFMS, $t \mathrm{BuOOH}, \mathrm{co}$-solvents as well as temperature (see Supplementary Information). The best conditions for the formation of $\mathbf{2 a}$ were found at $50 \mathrm{mM}$ phenol 1a with 2.0 eq. TFMS and 8.0 eq. $t \mathrm{BuOOH}$ at $30^{\circ} \mathrm{C}$ in the presence of $25 \mathrm{vol} \%$ dimethylsulfoxide (DMSO).

Functional group tolerance. To tap the scope and functional group tolerance of this method, various substituted phenols $\mathbf{~ 1 a - h}$ were transformed under optimized conditions, whereby the ortho- and para-position with respect to the phenolic hydroxyl moiety were blocked for substrate 1a-c (Table 2). In these cases, products $2 \mathrm{a}$-c with the $\mathrm{CF}_{3}$-moiety meta to the $\mathrm{OH}$ were isolated with exquisite regio-control (entry $1-3$ ), and for $\mathbf{2 b}$ and $\mathbf{2 c}$ also verified by X-ray crystallography (Fig. 3). Comparable results were obtained independent of the trifluoromethylation agent employed: thus, the Langlois' reagent as well as the Baran's zinc sulfinate led to comparable isolated yields up to $62 \%$. Moreover, the reaction system tolerated ketone-, ester-aldehyde, as well as nitrile-functionalities, emphasizing the mildness of the reaction. Interestingly, nitrogen-containing substrates like indol or 4 -aminoacetophenone were not converted at all under the reaction conditions investigated, while other substrates like

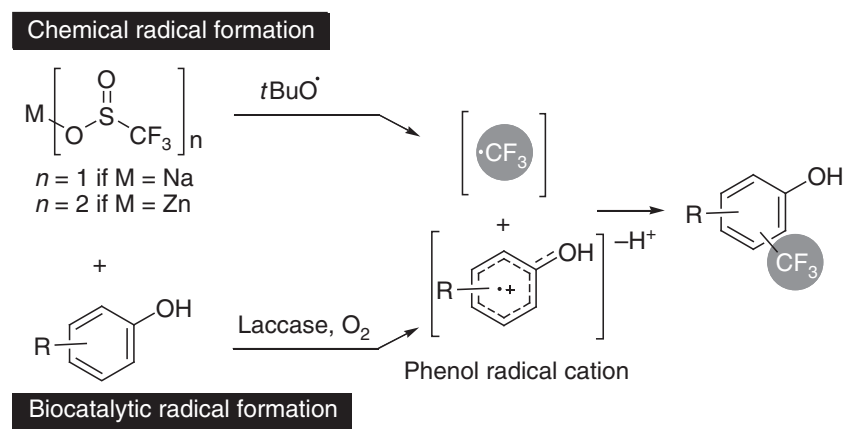

Figure 2 | Biocatalytic trifluoromethylation of unprotected phenols. Radical trifluoromethylation of unprotected phenols by recombination of radicals generated via two different pathways. 


\section{Table 1 | Laccase-mediated trifluoromethylation of unprotected phenols in combination with TFMS.}<smiles>COc1cc(C(C)=O)cc(OC)c1O</smiles>

$1 \mathrm{a}$$$
\text { (1) }
$$

Catalysts / reagents

$\mathrm{NaOAc}$ buffer (pH 5.5, $250 \mathrm{mM}$ ), $\mathrm{O}_{2}$ 25 vol\% DMSO, $30^{\circ} \mathrm{C}, 750$ r.p.m., 24 h<smiles>CCCCCCCC(=O)c1cc(OC)c(C(F)(F)F)c(OC)c1O</smiles>

2a

\begin{tabular}{llcc} 
Entry & Catalysts/reagents & Conv. (\%) & 2a (\%) \\
\hline 1 & Laccase $+\mathbf{1 a}+\mathrm{TFMS}+\mathrm{tBuOOH}$ & $99.4 \pm 0.2$ & $58.0 \pm 1.7$ \\
2 & TFMS + tBuOOH $+\mathbf{1 a}$ & n.c. & - \\
3 & Laccase $+\mathbf{1 a}$ & $77.1 \pm 0.3$ & $<0.1$ \\
4 & Laccase $+\mathbf{1 a}+\mathrm{TFMS}$ & $23.6 \pm 0.8$ & $<0.1$ \\
5 & Laccase $+\mathbf{1 a}+\mathrm{TFMS}+\mathrm{H}_{2} \mathrm{O}_{2}$ & n.c. & - \\
\hline
\end{tabular}

Laccase, Agaricus bisporus laccase; n.c., no conversion;

TFMS $=\mathrm{Zn}\left(\mathrm{SO}_{2} \mathrm{CF}_{3}\right)_{2}=((($ trifluoromethyl) sulfinyl)oxy $)$ zinc salt.

${ }^{*}$ Based on recovered starting material.

† Determined by GC

sesamol, 5,6,7,8-tetrahydro-2-naphthol, 2-naphthol or metadimethylamino acetophenone resulted in complex product mixtures.

Regioselectivity. The transformation of phenols $\mathbf{1 d}$ and 1e, bearing only a single substituent ortho to the phenolic hydroxy group, led to a mixture of regio-isomers, albeit with significant preference for the meta-isomers $(\mathrm{C} 2: \mathrm{C} 3=4: 1$ up to $10: 1$, entry 4 and 5). The preferred meta-substitution for $\mathbf{2 d}$ was confirmed via a crystal structure (Fig. 3). It is worth noting that substrate 1f, being devoid of ortho-methoxy substituents, afforded only the isomer bearing the $\mathrm{CF}_{3}$ moiety ortho to the alcohol group with $31 \%$ isolated yield (entry 6). In a similar fashion, substrate $\mathbf{1 g}$ possessing in para position a nitrile group instead of the acetyl moiety resulted in the mono-substituted ortho-product $\mathbf{2 g}$ with $57 \%$ isolated yield (entry 7). Taking a substrate devoid of a para-substituent but having methoxy substituents in both ortho positions (1h), the trifluoromethylation protocol led to a di-trifluoromethylated product, $\mathbf{2 h}$, having a $\mathrm{CF}_{3}$-group in ortho as well as meta position with respect to the phenolic $\mathrm{OH}$ (entry 8).

The observed regioselectivity for $\mathbf{2 d - f}$ can be explained by the transition state energies of the addition of the $\mathrm{CF}_{3}$-radical to the phenol radical cations. For instance, the corresponding transition state leading to $\mathbf{2 d}$ with substitution at C2 is energetically preferred over substitution at C3 $\left(2.1 \mathrm{kcal} \mathrm{mol}^{-1}, \mathrm{M} 06-2 \mathrm{X} / 6-\right.$ $311+\mathrm{G}(\mathrm{d}, \mathrm{p}))$ (Fig. 4, Supplementary Fig. 5). The same is true for the analogous transition state leading to $2 \mathrm{e}$ (Supplementary Fig. 6). The energies of the substituted intermediate cations after addition of the $\mathrm{CF}_{3}$-radical to the phenol radical cation also reflect the observed regioselectivity. In the case of substrate 1f, the energies of the transition states support the expected and observed substitution in ortho-position to the phenolic $\mathrm{OH}$ leading to product $2 \mathrm{f}$ (Supplementary Fig. 6). Since the energies of the transition states reflect the observed regioselectivity, the bio-trifluoromethylation is mainly not active site-directed.

Mechanism. The computational calculations also showed that the $\mathrm{CF}_{3}$-radical has to react preferentially with the phenol radical cation and not with the corresponding already deprotonated phenoxy radical, since the latter would lead, for example, for 1d, to substitution in ortho-position to the hydroxy group and not at the mainly observed meta-position. Substitution in ortho-position

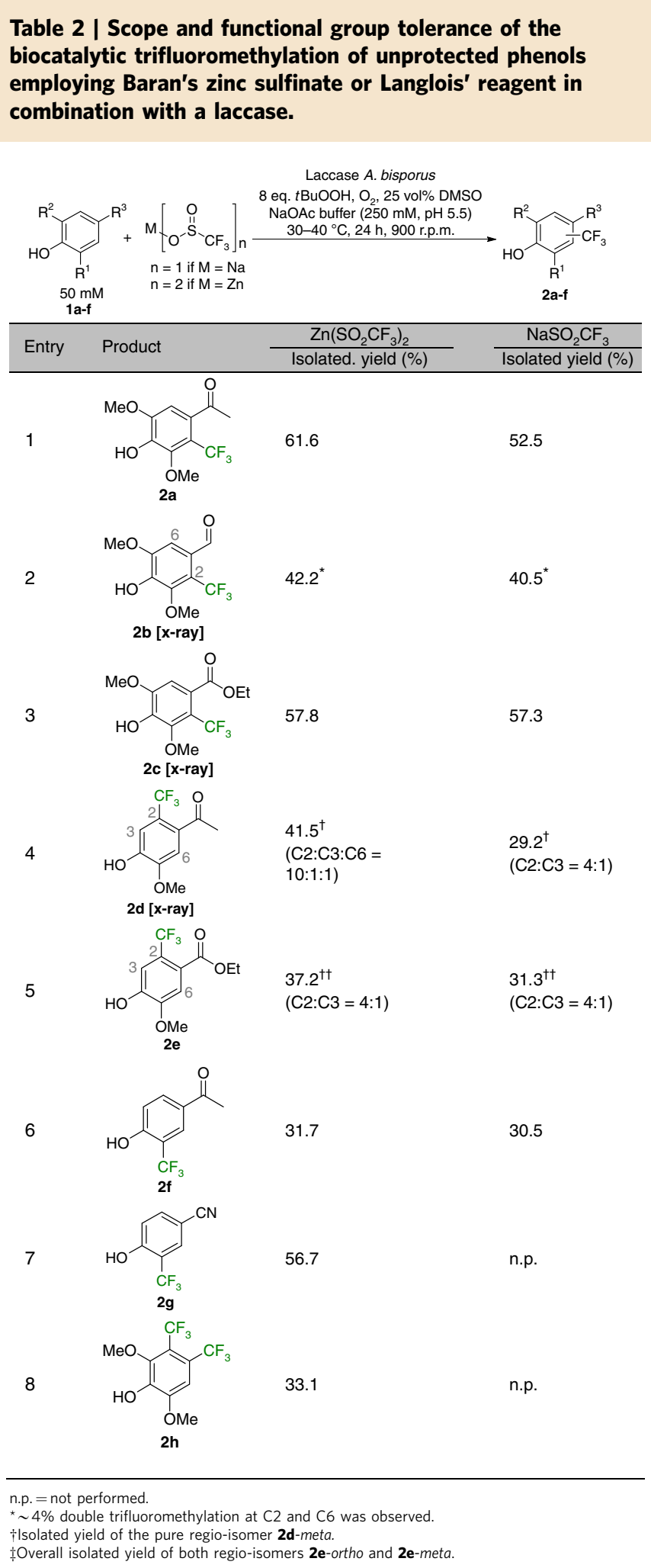

would be favoured over meta-position by $3 \mathrm{kcal} \mathrm{mol}^{-1}$ upon combination with the neutral phenoxy radical. Therefore, in the proposed mechanism the laccase oxidizes the phenol 1d via a single electron transfer to the phenol radical cation (Fig. 5). The latter reacts with the $\mathrm{CF}_{3}$-radical to give the cationic intermediate, which rearomatizes to the final product. As shown in the initial experiments, trifluoromethylation only occurred in the presence of laccase and TFMS/ $t \mathrm{BuOOH}$ (Table 1, entry 1); the phenol 
starting material did not react with TFMS/ $t \mathrm{BuOOH}$ (entry 2 ), nor did the phenol radical cation (formed by laccase and $\mathrm{O}_{2}$ present) react with TFMS (entry 4 ).

In the recently proposed mechanism ${ }^{39}$, traces of redox metals are proposed to initiate the reaction for the first transformation of $t \mathrm{BuOOH}$ to $t \mathrm{BuO} \bullet$ and $\mathrm{OH}^{-} . t \mathrm{BuO} \bullet$ enables the formation of the $\mathrm{CF}_{3} \bullet$ species. In the reported catalytic cycle the activation of $t \mathrm{BuOOH}$ was triggered by the heteroaromatic radical intermediates.

Since in the laccase-catalysed trifluoromethylation of phenol, the laccase provides already one reactive radical species for the $\mathrm{C}-\mathrm{C}$ bond forming reaction, namely the phenol radical cation, stoichiometric amounts of redox metal (for example, $\mathrm{Fe}, \mathrm{Co}, \mathrm{Cu}$ mentioned in previous work) would be required to obtain the amount of $t \mathrm{BuO} \bullet$ needed. Since the trifluoromethylation went to high conversion without addition of any metals or other redox reagents, it was deduced that the copper $\mathrm{Cu}(\mathrm{I})$ present in the laccase also reacts with $t \mathrm{BuOOH}$ to give $t \mathrm{BuO} \bullet$ and $\mathrm{Cu}(\mathrm{II})$ as already proposed in previous papers using only $\mathrm{Cu}$ (ref. 31). This
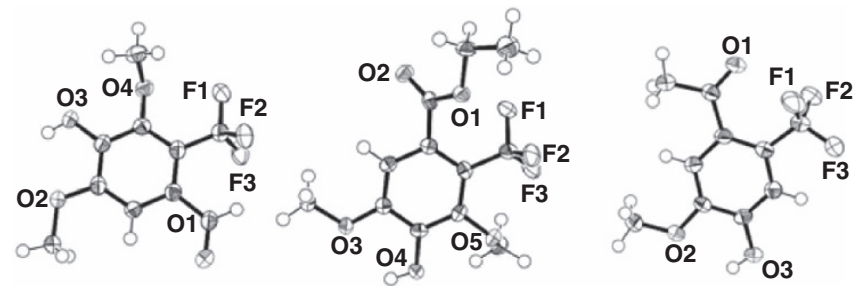

Figure 3 | Crystal structures of $\mathbf{2 b}, \mathbf{2 c}$ and $\mathbf{2 d - m e t a . ~ S t e r e o s c o p i c ~ O R T E P ~}$ plot of crystals $\mathbf{2 b}, \mathbf{2 c}$ and $\mathbf{2 d - m e t a . ~ T h e ~ p r o b a b i l i t y ~ e l l i p s o i d s ~ w e r e ~ d r a w n ~}$ on $50 \%$ probability. Radii of hydrogen atoms are drawn arbitrarily. was also supported by photometric assays in laccase-catalysed oxidative dimerization of 2,6-dimethoxy phenol showing that the presence of $t \mathrm{BuOOH}$ led to a faster reaction (Supplementary Methods, Photometric Enzymatic Activity Assay). Thus, the $\mathrm{Cu}(\mathrm{I})$ of the laccase can be oxidized by $t \mathrm{BuOOH}$, which leads to $t \mathrm{BuO} \bullet$ as previously reported ${ }^{31}$; the latter reacts with the $\mathrm{CF}_{3} \mathrm{SO}_{2}^{-}$to set free the $\mathrm{CF}_{3}$-radical, as proven elsewhere ${ }^{39}$.

Comparison to literature methods. To compare the herepresented laccase $/ \mathrm{BuOOH}$ protocol with published methods for the chemical trifluoromethylation of (electron-rich) arenes and hetero-arenes ${ }^{29,30}$, phenols $\mathbf{1 a}(\mathrm{R}=\mathrm{Me})$ and $\mathbf{1 b}(\mathrm{R}=\mathrm{H})$ were treated with Ruppert-Prakash reagent ${ }^{44} \mathrm{TMSCF}_{3}$ in the presence of catalytic silver (AgF) and $\mathrm{PhI}(\mathrm{OAc})_{2}$ as oxidant (Fig. 6a).

For both substrates $\mathbf{1 a}$ and $\mathbf{1 b}$ only minor amounts of $\mathbf{2 a}$ and 2b were found in a complex product mixture; the major product components were the trifluoromethyl-aryl-ethers $\mathbf{3 a}$ and $\mathbf{3 b}$ (13-17\% isolated yield). A related $\mathrm{CF}_{3}$-ether formation was reported recently 45 .

As a second literature method a metal-free alternative for the trifluoromethylation of arenes and biaryls was investigated ${ }^{30}$, whereby the $\mathrm{CF}_{3}$-radical is generated by the oxidation of the Langlois' reagent $\mathrm{NaSO}_{2} \mathrm{CF}_{3}$ with phenyl-iodine bis(trifluoroacetate) (Fig. 6b). In this case the transformation of $\mathbf{1 a}$ and $\mathbf{1 b}$ led to the corresponding trifluoromethanesulfonates $\mathbf{4 a}$ and $\mathbf{4 b}$ as the main products (18-89\% isolated yield), while $\mathbf{2 a}$ and $\mathbf{2 b}(\mathrm{R}=\mathrm{H})$ were found only in negligible quantities.

As a third method the Togni reagent ${ }^{32,46}$ was employed for substrate 1a (Fig. 6c); although the substrate was completely converted, product $\mathbf{2 a}$ was only a minor product (8\%), while two non-identified main products were detected, which did not contain any $\mathrm{CF}_{3}$-group.
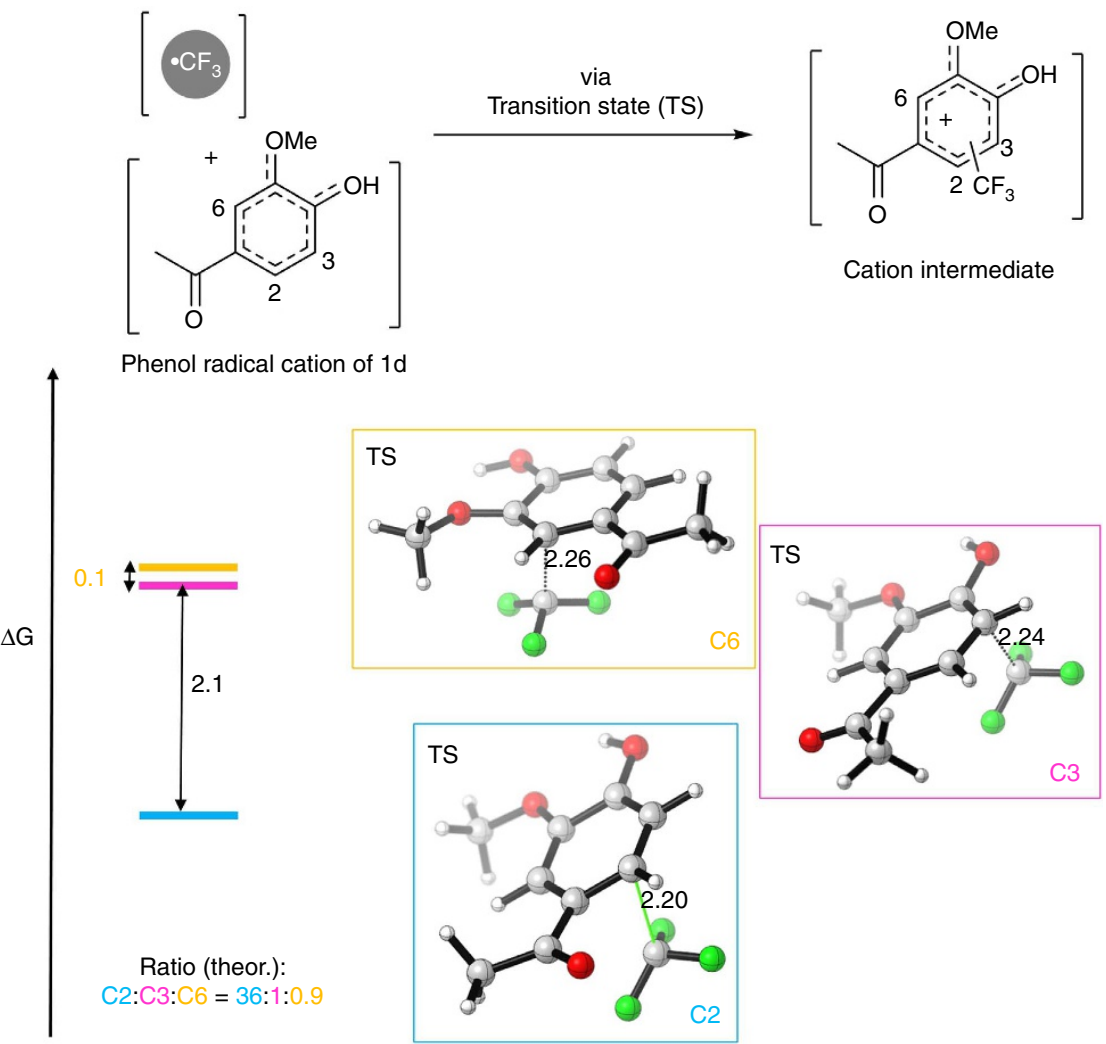

Figure 4 | Energy differences of transition states. Energy differences and structures of transition states leading preferentially to substitution at C2 giving $\mathbf{2 d}$ 


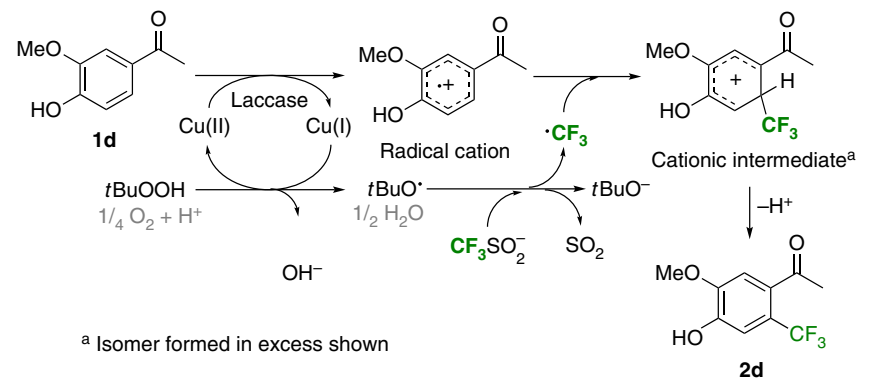

Figure 5 | Proposed mechanism for laccase-catalysed trifluoromethylation of phenols. Proposed mechanism for the laccase-mediated trifluoromethylation of unprotected phenols exemplified for substrate 1d. For reasons of clarity the scheme displays only the productive pathway relevant for the formation of $\mathbf{2} \mathbf{b}$; other reactions, for example, like $\mathrm{HCF}_{3}$ formation, dimerization of the $\mathrm{CF}_{3}$-radical or the radical cation, the decay of $t \mathrm{BuOOH}$ and the oxidation of $\mathrm{SO}_{2}$ are omitted, as well as the formation of minor regio-isomers.

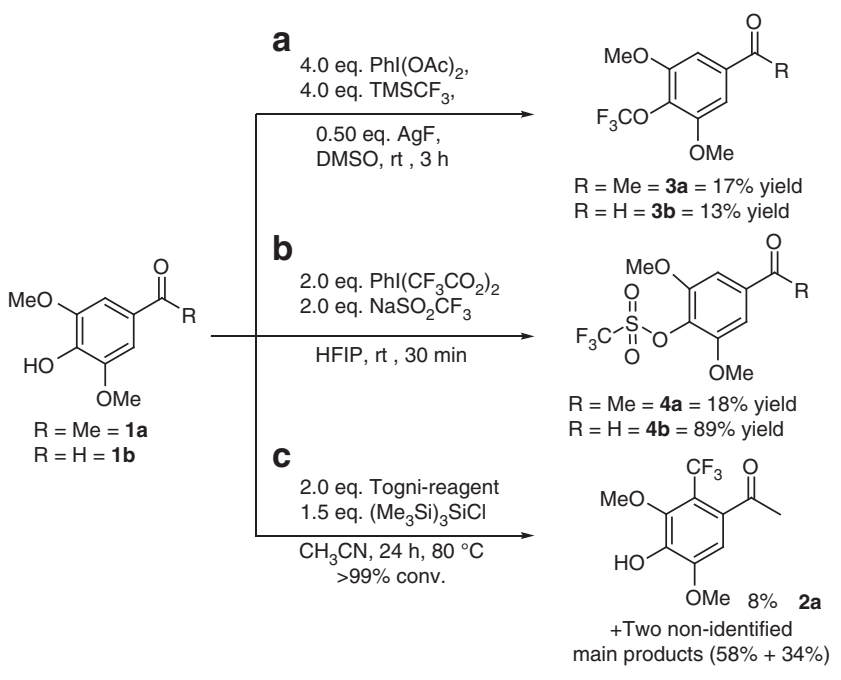

Figure 6 | Methods from literature tested for comparison.

Trifluoromethylation methods for electron-rich arenes for comparison with the here-presented laccase/tBuOOH concept. (a) Method involving silver as metal; metal-free trifluoromethylations using phenyl-iodine bis(trifluoroacetate) (b) or Togni-reagent (c).

Thus, the laccase trifluoromethylation reported here is clearly complementary to literature methods tested.

\section{Discussion}

With this study, we have achieved radical $\mathrm{C}-\mathrm{CF}_{3}$ bond formation by the recombination of two radical species-one generated biocatalytically and the other in a chemical reaction. This method represents the first biocatalyst-dependent trifluoromethylation of organic compounds, especially unprotected phenols, giving access to building blocks that were not accessible as major products by any other method described before. Moreover, the methods display a high functional group tolerance, allowing the conversion of aldehydes, esters and ketones without decomposition, which makes this method suitable for late-stage trifluoromethylations. The method proceeds under mild reaction conditions with high regioselectivity.

\section{Methods}

Representative trifluoromethylation procedure $(1 \mathrm{ml})$. The laccase from $A$. bisporus (7.5 U, $5.0 \mathrm{mg} \mathrm{ml}^{-1}$ final conc.) was dissolved in a sodium acetate buffer (695 $\mu \mathrm{l}, 250 \mathrm{mM}$, pH 5.5) prior to addition of $\mathrm{Zn}\left(\mathrm{SO}_{2} \mathrm{CF}_{3}\right)_{2}$ (2 eq., $33.2 \mathrm{mg}$ dissolved in DMSO). Afterwards ketone 1 ( $50 \mathrm{mM}$ final concentration, dissolved in DMSO) was added followed by aqueous $t \mathrm{BuOOH}$ solution ( 8.0 eq., $55 \mu \mathrm{l}$, $70 \mathrm{wt} \%$ aqueous solution) to reach a total volume of $1.0 \mathrm{ml}$ (25 vol\% DMSO). The reactions were shaken in an orbital shaker at $30^{\circ} \mathrm{C}\left(\mathrm{Zn}\left(\mathrm{SO}_{2} \mathrm{CF}_{3}\right)_{2}\right)$ or $40^{\circ} \mathrm{C}$ in case of $\mathrm{NaSO}_{2} \mathrm{CF}_{3}$ for $24 \mathrm{~h}$ at 900 r.p.m. (horizontal position). Then, each $1 \mathrm{ml}$ reaction was extracted four times with EtOAc $(500 \mu \mathrm{l})$ and combined organic fractions were dried over $\mathrm{Na}_{2} \mathrm{SO}_{4}$. The solutions were filtered, concentrated under reduced pressure and the residue was purified by various solvent mixtures to afford the trifluoromethylated phenol derivative 2 .

QM calculations. Full geometry optimizations, transition structure searches and single-point computations were carried out with the Gaussian 09 package ${ }^{47}$. All geometry optimizations were carried out with the unrestricted version of the hybrid B3LYP functional ${ }^{48}$. For $\mathrm{C}, \mathrm{O}, \mathrm{N}$ and $\mathrm{H}$, the double-zeta basis set $6-31 \mathrm{G}(\mathrm{d})$ was employed to obtain the geometries, and the larger $6-311+\mathrm{G}(\mathrm{d}, \mathrm{p})$ basis set was used to calculate single-point energies. Additional single-point energy calculations using functionals able to account for dispersion forces such as M06-2X (ref. 49) in conjunction with the $6-311+\mathrm{G}(\mathrm{d}, \mathrm{p})$ basis set were performed (Supplementary Table 5). Thermal and entropic corrections to energy were calculated from vibrational frequencies. The nature of the stationary points was determined in each case according to the appropriate number of negative eigenvalues of the Hessian matrix from the frequency calculations. Frequencies were not scaled.

Data availability. Crystal structures that support the findings of this study have been deposited at the Cambridge Crystallographic Data Centre and allocated the deposition numbers CCDC 1480621 (2b), 1480623 (2c) and CCDC 1480622 (2d). All other data supporting the findings of this study are available within the article and its Supplementary Information file or from the author upon reasonable request.

\section{References}

1. Purser, S., Moore, P. R., Swallow, S. \& Gouverneur, V. Fluorine in medical chemistry. Chem. Soc. Rev. 37, 320-330 (2008).

2. Zhu, W. et al. Recent advances in the trifluoromethylation methology and new $\mathrm{CF}_{3}$-containing drugs. J. Fluorine Chem. 167, 37-54 (2014).

3. Böhm, H.-J. et al. Fluorine in medicinal chemistry. ChemBioChem 5, 637-643 (2004).

4. Studer, A. A 'Renaissance' in radical trifluoromethylation. Angew. Chem. Int. Ed. 51, 8950-8958 (2012).

5. Wu, X.-F., Neumann, H. \& Beller, M. Recent developments on the trifluoromethylation of (hetero)arens. Chem. Asian J. 7, 1744-1754 (2012).

6. Liang, T., Neumann, C. N. \& Ritter, T. Introduction of fluorine and fluroine-containing functional groups. Angew. Chem. Int. Ed. 52, 2-53 (2013).

7. Bizet, V., Besset, V., Ma, J.-A. \& Cahard, D. Recent progress in asymmetric fluorination and trifluoromethylation reactions. Curr. Top. Med. Chem. 14, 901-940 (2014).

8. Furuya, T., Kamlet, A. S. \& Ritter, T. Catalysis for fluorination and trifluoromethylation. Nature 473, 470-477 (2011).

9. Tomashenko, A. O. \& Grushin, V. V. Aromatic trifluoromethylation with metal complexes. Chem. Rev. 111, 4475-4521 (2011).

10. Liu, H., Gu, Z. \& Jiang, X. Direct trifluoromethylation of the C-H bond. Adv. Synth. Catal. 355, 617-626 (2013).

11. Cho, E. J. et al. The palladium-catalyzed trifluoromethylation of aryl chlorides. Science 328, 1679-1681 (2012).

12. Schareina, T. et al. Towards a practical and efficient copper-catalyzed trifluoromethylation of aryl halides. Top. Catal. 55, 426-431 (2012).

13. Morimoto, H., Tsubogo, T., Litvinas, N. D. \& Hartwig, J. F. A broadly applicable copper reagent for trifluoromethylations and perfluoroalkylations of aryl iodides and bromides. Angew. Chem. Int. Ed. 50, 3793-3798 (2011).

14. Ye, Y., Künzi, S. A. \& Sanford, M. S. Pratical method for the Cu-mediated trifluoromethylation of arylbornic acids with $\mathrm{CF}_{3}$ radicals derived from $\mathrm{NaSO}_{2} \mathrm{CF}_{3}$ and tert-butyl hydroperoxide. Org. Lett. 14, 4979-4981 (2012).

15. Xu, J. et al. Copper-catalyzed trifluoromethylation of aryl boronic acids using a $\mathrm{CF}_{3}^{+}$reagent. Chem. Commun. 47, 4300-4302 (2011).

16. Li, Y., Wu, L., Neumann, H. \& Beller, M. Copper-catalyzed trifluoromethylation of aryl- and vinylboronic acids with generation of $\mathrm{CF}_{3}$-radicals. Chem. Commun. 49, 2628-2630 (2013).

17. Khan, B. A., Buba, A. E. \& Gooßen, L. J. Oxidative trifluoromethylation of arylboronates with shelf-stable potassium (trifluoromethyl)trimethoxyborate. Chem. Eur. J 18, 1577-1581 (2012).

18. Litvinas, D. N., Fier, P. S. \& Hartwig, J. F. A general strategy for the perfluoroalkylation of arenes and arylbromides by using arylboronate esters and $[($ phen)CuRF]. Angew. Chem. Int. Ed. 51, 536-539 (2012). 
19. Dai, J.-J. et al. Copper-promoted Sandmeyer trifluoromethylation reaction. J. Am. Chem. Soc. 135, 8436-8439 (2013).

20. Wang, X. et al. Silver-mediated trifluoromethylation of aryldiazonium salts: conversion of amino group into trifluoromethyl group. J. Am. Chem. Soc. 135, 10330-10333 (2013).

21. Wang, X., Truesdale, L. \& Yu, J.-Q. Pd(II)-catalyzed ortho-trifluoromethylation of arenes using TFA as a promoter. J. Am. Chem. Soc. 132, 3648-3649 (2010).

22. Zhang, X.-G., Dai, H.-X., Wasa, M. \& Yu, J.-Q. Pd(II)-catalyzed trifluoromethylation of arenes and insights into the coordinatation mode of acidic amide directing groups. J. Am. Chem. Soc. 134, 11948-11951 (2012).

23. Hafner, A. \& Bräse, S. ortho-Trifluoromethylation of functionalized aromatic triazenes. Angew. Chem. Int. Ed. 51, 3713-3715 (2012).

24. Xie, J., Yuan, X., Abdukader, A., Zhu, C. \& Ma, J. Visible-light promoted radical C-H trifluoromethylation of free anilines. Org. Lett. 16, 1768-1771 (2014).

25. Nagib, D. A. \& MacMillan, D. W. C. Trifluoromethylation of arenes and hetereoarenes by means of phororedox catalysis. Nature 480, 224-228 (2011).

26. Ye, Y. \& Sanford, M. S. Merging visible-light photocatalysis and transitionmetal catalysis in the copper-catalzyed trifluoromethylation of boronic acids with $\mathrm{CF}_{3} \mathrm{I}$. J. Am. Chem. Soc. 134, 9034-9037 (2012).

27. Mejia, E. \& Togni, A. Rhenium-catalyzed trifluoromethylation of arenes and hetereoarenes by hypervalent iodine reagents. ACS Catal. 2, 521-527 (2012)

28. Ye, Y., Lee, S. H. \& Sanford, M. S. Silver-mediated trifluoromethylation of arenes using $\mathrm{TMSCF}_{3}$. Org. Lett. 13, 5464-5467 (2011).

29. Seo, S., Taylor, J. B. \& Greany, M. F. Silver-catalysed trifluoromethylation of arenes at room temperature. Chem. Commun. 49, 6385-6387 (2013).

30. Yang, Y.-D., Iwamoto, K., Tokunaga, E. \& Shibata, N. Transition-metal-free oxidative trifluoromethylation of unsymmetrical biaryl with trifluoromethansulfinate. Chem. Commun. 49, 5510-5512 (2013).

31. Langlois, B. R., Laurent, E. \& Roidot, N. Trifluoromethylation of aromatic compounds with sodium trifluoromethanesulfinate under oxidative conditions. Tetrahedron Lett. 32, 7525-7528 (1991).

32. Stanek, K., Koller, R \& Togni, A. Reactivity of a 10-I-3 hypervalent iodine trifluoromethylation reagent with phenols. J. Org. Chem. 73, 7678-7685 (2008).

33. Yang, J.-J., Kirchmeier, R. L. \& Shreeve, J. M. New electrophilic trifluoromethylating agents. J. Org. Chem. 63, 2656-2660 (1998).

34. Riva, S. Laccases: blue enzymes for green chemistry. Trends Biotechnol. 24, 219-226 (2006).

35. Mogharabi, M. \& Faramarazi, M. A. Laccase and laccase-mediated systems in the synthesis of organic compounds. Adv. Synth. Catal. 356, 897-927 (2014)

36. Herter, S. \& Turner, N. J. Oxidation other than with cytochrome P450s. Sci. Synth. 3, 65-114 (2015).

37. Witayakrana, S. \& Ragauskas, A. J. Synthetic applications of laccase in green chemistry. Adv. Synth. Catal. 351, 1187-1209 (2009).

38. Zhou, H., Qiu, X., Yang, D. \& Xie, S. Laccase and xylanase incubation enhanced the sulfomethylation reactivity of alkali lignin. ACS Sustainable Chem. Eng. 4, 1248-1254 (2016).

39. Ji, Y. et al. Innate C-H trifluoromethylation of hetereocycles. Proc. Natl Acad. Sci. USA 108, 14411-14415 (2013).

40. Zhang, C. Application of Langlois' reagent in trifluoromethylation reactions. Adv. Synth. Catal. 356, 2895-2906 (2014).

41. Fujiwara, Y. et al. Pratical and innate carbon-hydrogen functionalization of heterocycles. Nature 492, 95-99 (2011).

42. O'Brien, A. G. et al. Radical C-H functionalization of heteroarenes under electrochemical control. Angew. Chem. Int. Ed. 53, 11868-11871 (2014).

43. Engelmann, C., Illner, S. \& Kragl, U. Laccase initiated C-C couplings: various techniques for reaction monitoring. Process Biochem. 50, 1591-1599 (2015).
44. Prakash, G. K. S. \& Yudin, A. K. Perfluoroalkylation with organosilicon reagents. Chem. Rev. 97, 757-786 (1997).

45. Liu, J.-B. et al. Silver-mediated oxidative trifluoromethylation of phenols: direct synthesis of aryl trifluoromethyl ethers. Angew. Chem. Int. Ed. 54, 11839-11842 (2015).

46. Wiehn, M. S., Vinogradova, E. V. \& Togni, A. Electrophilic trifluoromethylation of arenes and $\mathrm{N}$-heteroarenes using hypervalent iodine reagents. J. Fluorine Chem. 131, 951-957 (2010).

47. Frisch, M. J. et al. Gaussian 09 (Gaussian, 2009).

48. Lee, C., Yang, W. \& Parr, R. G. Development of the Colle-Salvetti correlationenergy formula into a functional of the electron density. Phys. Rev. B 37, 785-789 (1988)

49. Zhao, Y. \& Truhlar, D. The M06 suite of density functionals for main group thermochemistry, thermochemical kinetics, noncovalent interactions, excited states, and transition elements: two new functionals and systematic testing of four M06-class functionals and 12 other functionals. Theor. Chem. Acc. 120, 215-241 (2008).

\section{Acknowledgements}

E.B. received funding from the European Commission by a Marie Curie Actions-IntraEuropean Fellowship (IEF) in the project 'BIOCASCADE' (FP7-PEOPLE-2011-IEF)

N.R. has been supported by the Austrian BMWFJ, BMVIT, SFG, Standortagentur Tirol and ZIT through the Austrian FFG-COMET-Funding Program. Support by NAWI Graz and COST Action CM1303 Systems Biocatalysis is acknowledged. V.R. thanks the Austrian Science Fund (FWF) for an 'Erwin-Schroedinger' Fellowship (J3292).

\section{Author contributions}

R.C.S. and W.K. conceived, designed and supervised the project. E.B. performed chemical control reactions, V.R. photometric assays, R.C.S. and N.R. analytical optimization studies. Preparative scale experiments and interpretation: R.C.S. and V.R. W.K. performed computational experiments. R.C.S., K.N.H. and W.K. wrote and edited the manuscript All authors discussed the results and commented on the manuscript.

\section{Additional information}

Supplementary Information accompanies this paper at http://www.nature.com/ naturecommunications

Competing financial interests: The authors declare no competing financial interests.

Reprints and permission information is available online at http://npg.nature.com/ reprintsandpermissions/

How to cite this article: Simon, R. C. et al. Biocatalytic trifluoromethylation of unprotected phenols. Nat. Commun. 7, 13323 doi: 10.1038/ncomms13323 (2016).

Publisher's note: Springer Nature remains neutral with regard to jurisdictional claims in published maps and institutional affiliations.

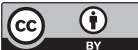

This work is licensed under a Creative Commons Attribution 4.0 International License. The images or other third party material in this article are included in the article's Creative Commons license, unless indicated otherwise in the credit line; if the material is not included under the Creative Commons license, users will need to obtain permission from the license holder to reproduce the material. To view a copy of this license, visit http://creativecommons.org/licenses/by/4.0/

C The Author(s) 2016 